Para enlazar con este artículo / To link to this article:

http://dx.doi.org/10.14198/fem.2018.32.11

Para citar este artículo / To cite this article:

Spairani Berrio, Silvia, Rosa Roca, Nuria y González Ponce, Eloísa. «¿Qué aporta la perspectiva de género a las intervenciones sostenibles en las edificaciones?». En Feminismo/s, 32 (diciembre 2018): 287-310. Dosier monográfico: MAS-MES: Mujeres, Arquitectura y Sostenibilidad - Medioambiental, Económica y Social, coord. María-Elia Gutiérrez-Mozo, DOI: 10.14198/fem.2018.32.11

\title{
¿QUÉ APORTA LA PERSPECTIVA DE GÉNERO A LAS INTERVENCIONES SOSTENIBLES EN LAS EDIFICACIONES?
}

\author{
WHAT BRINGS THE GENDER PERSPECTIVE \\ TO SUSTAINABLE INTERVENTIONS IN BUILDINGS?
}

Silvia SPAIRANI BERRIO

Universidad de Alicante silvia.spairani@ua.es orcid.org/0000-0002-8929-0993

Nuria RosA ROCA Universidad Católica de Murcia nrosa@ucam.edu orcid.org/0000-0001-7325-9206

Eloísa GONZÁLEZ PONCE Universidad Católica de Murcia egonzalez@ucam.edu orcid.org/0000-0002-9933-1488

\section{Resumen}

¿Cuál es el significado de una intervención sostenible con perspectiva de género, en lo que a edificación se refiere? ¿Cómo afrontar rehabilitaciones y restauraciones que tengan en cuenta la perspectiva de género? El objetivo de este artículo es reflexionar sobre las intervenciones sostenibles en el conjunto de edificaciones, teniendo en cuenta las complejas relaciones entre la educación universitaria, el empleo y el bienestar social, con el objetivo de impedir que la ideología de género quede aplazada, una vez más, a los artífices de la cohesión y de la armonía social. Todo ello, con el 
convencimiento del relevante e indiscutible papel desempeñado por la mujer en el ámbito de la construcción, aun adquiriendo éste un carácter no visible debido a la dificultad por la que la mujer atraviesa durante su inserción laboral en las áreas de la rehabilitación y la restauración. Este estudio se ha basado en una encuesta anónima realizada a una muestra de 37 estudiantes del Máster de Patología e Intervención en la Edificación de la Universidad Católica de Murcia, con el fin de conocer su opinión sobre si dichos estudios de posgrado han favorecido su inserción laboral desde el punto de vista de la paridad. Los datos obtenidos reflejan la existencia de diferencias entre sexos, durante los primeros años de incorporación de los/as egresados/as al mundo laboral en el ámbito de la intervención sostenible. De la misma manera, son diferentes las motivaciones y expectativas que pueden condicionar el desarrollo diferenciado por género, desde el punto de vista de la transformación de la realidad que poseen el diseño y la arquitectura sostenible.

Palabras clave: rol de género, inserción laboral, desarrollo de carrera, liderazgo transformacional.

\begin{abstract}
What do we mean by sustainable intervention in the building sector from a gender perspective? How to deal with rehabilitations and restorations of buildings taking the gender perspective into account? The aim of the paper is to discuss sustainable interventions in buildings, considering the complex relationships between college education, employment and social welfare, to prevent that the goal of the gender ideology is postponed once again to the architects of cohesion and social harmony. All of this, in the firm conviction of the relevant role-played by women the field of construction, despite it does not have visibility, due to the difficulty of the labor market insertion of women in rehabilitation and restoration fields. The study was based on an anonymous survey given to a sample of 37 students of the Master of Pathology and Intervention in the Building of the Catholic University of Murcia to know their opinion about if this postgraduate degree has encouraged their inclusion into employment from a gender equality perspective. According to the data obtained, there are differences between women and men during the early years of insertion into labor market around sustainable intervention. Moreover, motivations and expectations that can influence the development of differentiated by gender, from the point of view of the transformation of reality that have design and sustainable architecture are different.
\end{abstract}

Keywords: Gender role, labour insertion, career development, transformational leadership.

Feminismo/s 32, diciembre 2018, pp. 287-310 


\section{INTRODUCCIÓN: LOS DESEQUILIBRIOS DE GÉNERO EN LAS INTERVENCIONES O CONSERVACIONES SOSTENIBLES EN LAS EDIFICACIONES}

¿Qué significa una intervención sostenible en edificación con perspectiva de género? ¿Cómo afrontar rehabilitaciones y restauraciones que tengan en cuenta la perspectiva de género? El presente artículo nace de las inquietudes compartidas por las personas que realizamos esta investigación, ante los retos y complejidades que plantea el actual contexto de crisis sistémica (económica, social, ecológica y ética), el cual, sin duda, está agudizando las segregaciones ocupacionales entre los participantes en el sector de la construcción (Gil 62-63). Por desgracia, esta arraigada segregación horizontal perdura hasta la actualidad debido a la desigual ocupación de las mujeres respecto a los hombres, dado que se asocia a una distribución por sectores de actividad. De hecho, el sector se caracteriza por que la mujer tiene unos índices de representación mínimos, apenas 141.100 mujeres ocupadas en el sector en 2010 (I.F. de 9,34\%) frente a 1.510.300 hombres. En el año 2017 la tasa empeora, si cabe, debido al alto porcentaje de paro, únicamente 99.100 mujeres (I.F. de $9,62 \%$ ) respecto a los 1.029.200 hombres. Por tanto, sigue siendo a día de hoy el sector más masculinizado en comparación con el sector industrial, 660.600 mujeres (I.F. de 33,25\%) frente a 1.986 .800 hombres. En cambio, en los sectores agrícola, ganadero, de silvicultura y de pesca, nos encontramos con 194.800 mujeres (I.F. 31,18\%) frente a 624.700 hombres y, por último, en el sector servicios las cifran oscilan entre 7.604 .000 mujeres (I.F. 114,77\%) y 6.625.600 hombres. En este sentido, la evolución de la mujer que accede a un puesto de trabajo ha sido progresiva y ha tenido un comportamiento dinámico al alza en el sector de la construcción, pero insuficiente debido a la situación discriminatoria y de subordinación. Asimismo, la segregación vertical de género se manifiesta en dicho ámbito respecto de los niveles ocupacionales desempeñados por unos y otras, explicada mediante el conocido «techo de cristal». Al respecto, a finales del siglo XX, Fernández alude a este concepto desde el punto de vista del poder y el género:

El ejercicio histórico del poder ha creado una profunda convicción en la mayoría de los varones que los coloca automáticamente en una posición de mando y/o protección. Correlativamente con esto, las mujeres, al mismo tiempo que han legitimado y legitiman este poder de los varones, han ido

Feminismo/s 32, diciembre 2018, pp. 287-310 
ocupando nuevos espacios y han desarrollado en los intersticios de dicho poder variadas formas de resistencias, transgresiones y contra violencias que si bien no han revertido su situación de subordinación han ido conformando espacios sociales y subjetivos de significación. Si bien esto no establece de por sí la paridad, crea condiciones para que sea posible. (112)

Pero ¿por qué no existe paridad en el «techo de cristal» en el ámbito de la construcción? La conclusión de Barberá Ribera, Estellés Miguel y Dema Pérez a principios del siglo XXI, nos puede abrir líneas de reflexión a considerar:

[...] en el momento actual, el techo de cristal lo apuntalan dos consistentes pilares referidos a la cultura organizacional dominante, caracterizada por la persistencia de creencias sociales estereotipadas sobre los géneros, y a las responsabilidades asumidas mayoritariamente por las mujeres. (140)

La tasa de ocupación en relación a los perfiles profesionales relativos al ámbito de la construcción sigue siendo, hoy por hoy, un problema sin resolver que perjudica no sólo a las disciplinas del Diseño, la Arquitectura y el Urbanismo, sino a todas las disciplinas existentes en la sociedad en general. En contra de lo que debería esperarse, por la actual crisis laboral, la tasa de ocupación femenina estudiada del INE, $4 .^{\circ}$ trimestre, en el periodo 2006-2017, nos demuestra que en la sociedad española se está experimentando un crecimiento dinámico de ocupación femenina, dado que hemos pasado de un valor del 48,45\% al $53,24 \%$. Por ello, la igualdad real entre los sexos aún no está visibilizada. De hecho, al analizar la tasa nacional masculina en 2017, vemos que da un valor de 64,73\%, quedando todavía muy alejada de la femenina. Por lo tanto, desafortunadamente, a día de hoy podemos decir que las tasas de actividad masculina siempre superan a las femeninas.

A pesar de ello, el incremento de la participación femenina en el mercado laboral español sigue avanzando y es ya un hecho la feminización de este. Desde esta perspectiva, si observamos las tasas de actividad femenina del INE, 4. ${ }^{\circ}$ trimestre, teniendo en cuenta la edad, surgen discrepancias que debemos considerar y evaluar. Respecto a 2017, en la franja de edad de los 25 a 29 años, arroja datos destacables y alentadores, ya que la diferencia cuantitativa de tasa de actividad femenina es escasamente de un 3,67\% inferior a la masculina. Sin embargo, es lamentable que, durante el mismo año, en la franja de edad de los 30 a los 44 años, el porcentaje se triplique o cuadriplique, llegando a diferencias del 9,13\%, entre los 30 y los 34 años; del 9,34\%, entre 35 y 39

Feminismo/s 32, diciembre 2018, pp. 287-310 
años; de una diferencia del 8,97\%, de 40 a 44 años; de un 11,36\%, entre los 45 a los 49 años, y de un 15,35\%, de 50 a 54 años. La máxima diferencia para las mujeres es del 18,64\% y se alcanza en el tramo de edad de los 55 a los 59 años. Esta realidad es, en gran parte, consecuencia y reflejo de las barreras sociales que influyen en los procesos de inserción laboral de las mujeres. En general, los primeros periodos son coincidentes con la etapa en la que las mujeres suelen ser madres y adquieren más responsabilidades familiares, lo que significa que laboralmente las mujeres se encuentran en desventaja con respecto a la tasa de empleo masculino, a pesar del aumento en su nivel de formación. Con respecto a los tramos de edades desde el año 2006 hasta el 2017, donde más se aprecia la diferencia de tasa de actividad femenina es de los 16 a 19 años y de los 60 a los 64 años, pasándose del 24,78\% de ocupación, al 12,42\%, en el primer caso, del $21,57 \%$ al 38,55\%, en el segundo. En cambio, para edades comprendidas entre los 65 y los 69 años, el porcentaje de la tasa de ocupación apenas varía.

Por tanto, y aun no siendo todavía suficiente, la presencia progresiva de mujeres en el ámbito laboral, tanto de manera privada como pública, nos remite a una transformación cultural y social positiva que refleja el nuevo papel de la mujer contemporánea que accede al sector de la construcción. Según Infante, Román y Traverso:

Todo esto se puede ver acentuado en el sector de la construcción por el hecho de que los agentes implicados en el mercado laboral pudieran considerar a las mujeres como un colectivo único e invariable y que no están capacitadas para determinados trabajos. Por ejemplo, pudiera considerarse que las mujeres no pueden desempeñar el trabajo a pie de obra al no contar con suficiente fuerza física o preparación o motivación y que, además, pueden dar pie a situaciones de conflicto con los compañeros por rivalidades de tipo sexual o por hacerles asumir las tareas que ellas no sean capaces de hacer, y cuando alguna demuestra lo contrario lo consideran como la excepción que confirma la regla. (3-4)

De ahí la necesidad de un cambio de percepción en la sociedad respecto a las ventajas del empoderamiento de las mujeres. Dado que ello permitirá una visible movilidad femenina ascendente en los niveles jerárquicos del sector de la construcción, que incluya entre otros ámbitos laborales el conocimiento 
en las intervenciones sostenibles. Marta Lamas, respecto al cambio de percepción, indica:

[...] la posibilidad de pasar de un tipo de percepción a otro, de ampliar la mirada. La invisibilidad de cierto rasgo cultural se hace evidente al posicionarse en otro ámbito. Por ello, al percibir nuevas cuestiones, es posible efectuar un proceso transformativo. Si la gente cambia de forma rutinaria la base de su percepción, crea órdenes, escalas, niveles. El hecho de cambiar de perspectiva introduce posibilidades epistemológicas distintas. (330)

Esta afirmación implica, sin duda, concebir la realidad con una mirada más abierta a la igualdad de género. Es más, aunque hoy en día las mujeres son mayoría entre el alumnado universitario, tanto en grado como en posgrado, acabando también con mejores expedientes académicos, en promedio, que los de sus compañeros, el ámbito laboral de la intervención sostenible se resiste todavía a la participación femenina, aun habiendo adquirido las habilidades y conocimientos necesarios para cumplir con su perfil profesional. Desafortunadamente, esta realidad está marcada por la teoría del rol social, como indican López, Lisbona y Sáinz:

[...] la teoría predice que esas creencias sobre las características diferenciales de hombres y mujeres fomentan las conductas que confirman las expectativas en unas y en otros y así se reproducen los roles diferenciados por sexos. De este modo, se formaría un círculo vicioso difícil de romper: por una parte, para que cambien los aspectos descriptivos de los estereotipos se tienen que dar cambios sociales generales que incluyan cambios en la distribución de hombres y mujeres en los roles, por otra, los roles no cambian hasta que no cambien las creencias descriptivas que los sostienen. (163)

Los avances claros en el rol social de la mujer y su apuesta por estudios universitarios como el del sector de la construcción hacen que encontrar a las mujeres en puestos de trabajo en las obras deje de ser una anécdota. Sin embargo, las mujeres cuando por fin terminan y se van a incorporar laboralmente, sufren graves estereotipos descriptivos sobre rasgos de personalidad para justificar los obstáculos y conseguir un puesto de responsabilidad en la obra. ¿Realmente las estudiantes de ingenierías y arquitectura tienen conciencia de los obstáculos laborales a los que se deberán enfrentar? Navarro, Román e Infante lo tienen muy claro:

Feminismo/s 32, diciembre 2018, pp. 287-310 
Los estudios internacionales sobre barreras percibidas apuntan a una percepción del sector bastante cercana a la realidad, por parte de quienes aún no tienen experiencia en él. En la mayoría de las investigaciones, las estudiantes tienen consciencia de los obstáculos que les esperan en el mercado laboral; no obstante, la asignación sexual de los puestos y funciones, así como la exclusión de las mujeres de las redes sociales, son dos barreras no identificadas como tales. Merece mención el caso de aquellas estudiantes que, sabiendo que existen, piensan que no les van a afectar. (115)

De este modo se evidencia que existen rasgos de personalidad y códigos de comunicación e interacción excluyentes para las mujeres que se manifiestan en el trato hacia ellas. Se observa que la percepción desde el ámbito social, cultural y laboral no es neutral, soslayando situaciones difíciles para las arquitectas e ingenieras más jóvenes que ahora empiezan su andadura profesional, rara vez consideradas iguales entre sus compañeros varones de trabajo y constantemente interiorizadas por sus superiores y clientes. Ante estos últimos, la mujer topa con el absurdo deber de demostrar en reiteradas ocasiones una valía digna del puesto que ocupa; de ahí la necesidad inminente de romper con el sometimiento femenino al que queda actualmente expuesta. López, Lisbona y Sáinz indican al respecto:

Se plantean los primeros años de vida profesional como una etapa de lucha, en un mercado de trabajo muy competitivo, hasta que consigan consolidar un determinado puesto [...] No obstante, el miedo a la discriminación no se centra solamente en la empresa, sino en la imagen que hay que dar de cara a los clientes y en los estereotipos sociales que persisten sobre los roles de género y las ingenierías. (176)

Podemos observar que el tema más preocupante en este contexto no es sólo la discriminación de desventaja de la mujer, frecuentemente percibida como normal, sino también el modelo social ancestral referente a los desequilibrios de género en su actuación laboral con respecto a las intervenciones sostenibles, desde la disciplina del Diseño y la Arquitectura.

Estas inquietudes nos han conducido a reflexionar sobre la compleja relación existente entre los individuos afines a la construcción, así como sobre los estudios universitarios conexos a dicha temática y su repercusión en el mercado de trabajo y, por tanto, a adentrarnos en el proceso que suponen las intervenciones sostenibles desde el punto de vista óptimo del desarrollo vital y profesional de las mujeres que lo protagonizan, y las exclusiones a las

Feminismo/s 32, diciembre 2018, pp. 287-310 
que se ven expuestas para poder constatar la diferencia existente entre lo que debiera ser y lo que es en realidad. Una mirada crítica que refleje las distintas formas que han adoptado la desigualdad y las relaciones laborales de poder en el ámbito de la construcción, en función del género, y que en no pocas ocasiones derivan en el veto del acceso al «techo de cristal» en este sector.

Es necesario que los desequilibrios de género desde el punto de vista de las intervenciones sostenibles potencien el desarrollo de una ciudadanía global, crítica y comprometida con el diseño y la arquitectura sostenible, y que permitan visibilizar y empoderar a la mujer en estos campos. En palabras de Barahona, Gratacós y Quintana:

Conciencia de la integralidad de la persona humana, y de su dignidad más allá del mercado: la perspectiva de una ciudadanía global plantea un desarrollo centrado en el ser humano integral, caracterizado, por encima de todas las cosas, por su capacidad crítica para desvelar la realidad y reconocer la diferencia entre lo que 'es' y lo que 'debería ser'. Consecuentemente, reconoce que ese 'debería ser' se ha de construir desde una doble perspectiva: primero, desde un diálogo participativo en el que nadie imponga su visión sobre las otras personas; $y$, segundo, desde una consciencia histórico-política de implicarse en la construcción de una sociedad más justa, más libre y más solidaria, que beneficie sobre todo a las mayorías excluidas en cada contexto y en el entorno global. (14)

En la actualidad, existen algunas aportaciones españolas relevantes sobre temas del sector de la construcción (Infante, Román y Traverso, «El sector español de la construcción 32-43; Infante, Román y Traverso, «La educación universitaria» 1-11; Navarro, Román e Infante 103-117; Román, Ríos y Traverso 87-99; López, Lisbona y Sáinz 161-180). Sin embargo, no hacen alusión explícita al sector de la conservación y restauración del patrimonio edificado. En consecuencia, echamos en falta la existencia de trabajos que analicen y recojan de forma sistemática todo lo que gira en torno a las mujeres y su relación con las intervenciones sostenibles, tendentes a la erradicación de las desigualdades que someten y oprimen a las mujeres en el ámbito de la construcción, entre otros.

Este artículo pretende ser una contribución en este sentido para ayudar a la formación de los agentes intervinientes en dicho ámbito, involucrándolos en el desarrollo de una sociedad más justa e igualitaria en la constitución de las identidades de mujeres y hombres, propiciando por tanto el cambio y

Feminismo/s 32, diciembre 2018, pp. 287-310 
la transformación de las desigualdades. Para ello, analizamos una encuesta anónima realizada a una muestra de 37 estudiantes del Máster citado, desde el curso 2012-2013 al curso 2016-2017, en la Universidad Católica de Murcia, con el fin de conocer su opinión sobre si dichos estudios de posgrado han favorecido su inserción laboral desde el punto de vista de la paridad.

Por tanto, pensamos que la encuesta es de gran utilidad al estudio de este artículo para profundizar en el análisis del quehacer investigativo que se posee al respecto en estos momentos de cambios continuos y generalizados, en un primer acercamiento a los desequilibrios de género en las intervenciones sostenibles y, especialmente, explicado a través de la voz de los/as participantes egresados/as en el ámbito de la edificación, en la citada asignatura, para ver la situación de las mujeres frente al empleo.

Las reflexiones previas sitúan el tema central de este artículo en la importancia de la perspectiva de género desde el ámbito de la construcción y matizamos que, desde un punto de vista crítico, conviene poner en igualdad de condiciones las exigencias derivadas de las intervenciones sostenibles en el ámbito de la rehabilitación y la restauración, así como las derivadas de la sensibilización de agentes presentes en el proceso educativo.

El problema de fondo es que todo lo expuesto debe reflexionarse desde la premisa de que los derechos de todas las personas involucradas en la construcción son una cuestión de inclusión e igualdad de la ciudadanía y no de relaciones de poder hegemónicas y socialmente establecidas.

En nuestra opinión, todos estos factores no han sido suficientemente estudiados en el actual contexto de crisis sistémica (económica, social, ecológica y ética) en el que nos encontramos, y es el objeto de nuestra reflexión. Por ello, dichos factores requieren de un estudio y análisis más exhaustivos, incluyendo la lógica del cambio generacional de las mujeres dentro de un ámbito de trabajo tan marcadamente masculino que, sin duda, imposibilita el análisis del gran impulso de la innovación tecnológica desde el punto de vista de la equidad, mediante la adopción de normas y actuaciones que fomenten un cambio desde la disciplina del Diseño y la Arquitectura hacia los llamados edificios casi nulos, contribuyendo a la reducción de las emisiones de efecto invernadero, a raíz de mitigar el efecto del cambio climático Nearly Zero Energy Buildings (Comisión Europea, 2017), según DEEE, 2002/91/EC y 2010/31/EC (Parlamento Europeo y Consejo de la Unión Europea, 2010).

Feminismo/s 32, diciembre 2018, pp. 287-310 
Es aquí donde consideramos necesario el empoderamiento de la mujer, es decir, los cambios que se están produciendo en las ciudades derivan en la imposición de una serie de medidas y criterios de rehabilitación de edificios existentes, para darles un nuevo uso y reducir el consumo energético, provocando que la mujer se descubra a sí misma como un agente productivo y muy valioso desde el punto de vista de su aparición en el sector de las intervenciones sostenibles.

A continuación, y después de esta introducción, nos vamos a centrar en la descripción de los apartados de los que consta este artículo, cuyos contenidos se han articulado específicamente en torno a cinco secciones temáticas.

El primer bloque está dedicado a «Los desequilibrios de género en las intervenciones o conservaciones sostenibles en las edificaciones», sección en la que se desarrollan las reflexiones que han permitido establecer el marco teórico de este artículo.

Fruto de estas reflexiones, en la sección dedicada a «El forzoso aterrizaje de las mujeres en el sector de las intervenciones sostenibles en España» plasmamos una panorámica de la evolución del papel de la mujer en el sector de la construcción, centrada en la situación que presenta en los ámbitos laborales y educacionales. Para ello se analiza si existe segregación horizontal por sexo, tanto en el sector de la construcción como en las ramas de estudios universitarios. Asimismo, se aborda una serie de reflexiones que implican la puesta en marcha de unas pautas de actuación para optimizar la inserción laboral del colectivo femenino.

El bloque dedicado a «Metodologías y encuestas» se centra en los aspectos metodológicos de la investigación, tales como su diseño y planificación, con una encuesta anónima dirigida al alumnado del Máster de Patología e Intervención en la Edificación de la Universidad Católica de Murcia.

En la sección «Resultados» la encuesta se analiza con mucha cautela, pues sólo ha sido contestada por un grupo de alumnado de unos estudios concretos y aunque es posible que en otras áreas educativas universitarias los resultados cambien, sí son susceptibles de análisis, ya que juegan un papel importante para mostrar los grandes desequilibrios de género respecto a la proyección profesional del colectivo femenino en el ámbito de las intervenciones sostenibles en las edificaciones.

Feminismo/s 32, diciembre 2018, pp. 287-310 
Se concluye con la sección quinta «Conclusiones, limitaciones y futuras líneas de investigación en el trabajo». En ella se recogen las reflexiones que emanan de la praxis, tanto en la vertiente teórica como en la empírica, resaltando las implicaciones que aporta el empoderamiento de la perspectiva de género a las intervenciones sostenibles en las edificaciones, enumerando las limitaciones del estudio empírico desarrollado y proponiendo líneas de acción para optimizar la incorporación de la mujer a distintas esferas de la vida laboral en este sector.

\section{EL FORZOSO ATERRIZAJE DE LAS MUJERES EN EL SECTOR DE LAS INTERVENCIONES SOSTENIBLES EN ESPAÑA}

Se pone sobre la mesa la necesidad de dilucidar las características del modelo de desarrollo en el ámbito de la construcción actual, desde una perspectiva crítica de igualdad real y efectiva, y de cuestionar unas bases y fundamentos sujetos a la lógica de la equidad basada en un sistema sostenible más social, justo y equitativo desde una mirada feminista que plantee alternativas. No sólo porque se trate de aplicar la perspectiva de género, sino también porque la propia naturaleza de la adaptación a las normativas exige un análisis integral de intervención sostenible que permita la deconstrucción de los enfoques tradicionales de la edificación, muy influenciados por el avance tecnológico en las cuestiones relacionadas con las disciplinas del Diseño, la Arquitectura y el Urbanismo, y que han ignorado la participación de las mujeres hasta que han logrado formarse y prepararse para lo requerido en el mundo actual.

Es importante tener en cuenta el crucial asunto por el que surgen las preguntas iniciales: ¿Qué significa una intervención sostenible en edificación con perspectiva de género? ¿Cómo afrontar rehabilitaciones y restauraciones que tengan en cuenta la perspectiva de género? Estas preguntas, aparentemente sencillas, esconden tras de sí la necesidad de abordar con eficacia una perspectiva de género en las intervenciones sostenibles que fomente la mejora del estatus económico de las mujeres, el reconocimiento social y la eliminación de estereotipos educacionales. No es sólo por una cuestión de justicia, sino también por la necesidad urgente de hacer frente a las barreras estructurales para el logro de los derechos de las mujeres, con el fin de que esta evolución permita en el entorno urbano de las ciudades mantener las esencias del pasado

Feminismo/s 32, diciembre 2018, pp. 287-310 
y al mismo tiempo dejar constancia de nuestro paso por la historia, y que nuestra apuesta por una equidad de género llena de esperanzas y realidades se concentre en actuaciones relativas a la creación de entornos respetuosos con la diversidad humana, incluyendo el propio entorno de la rehabilitación y restauración de edificios, donde la pluralidad cultural, las diferentes formas de vida, el estilo de aprendizaje del individuo y las particularidades locales, se conviertan en elementos esenciales a preservar.

Desafortunadamente, el tipo de ocupación laboral marca a la mujer. En España, la Encuesta de la Población Activa del INE de 2017 nos muestra que, en las profesiones del sector de la construcción, las mujeres que han accedido a un empleo ocupan una proporción muy baja (1,16\% frente al 10,03\% de hombres), lo que marca una clara segregación ocupacional por sexos, muy alejada todavía de los parámetros de la igualdad efectiva. Por ello, un dato destacable y desalentador es que respecto a 2013 (1,11\% de ocupación femenina frente al 10,12\% de ocupación masculina), la brecha de género únicamente se haya reducido en $0,05 \%$, factor que sin duda ha venido marcado por existir todavía a día de hoy una gran crisis en este sector.

La feminización del trabajo en la construcción tiende actualmente a evolucionar favorablemente, aunque de manera muy lenta. Así pues, en una primera impresión podría parecer que la gran incorporación de la mujer a una titulación de educación superior en los últimos años favorecería enormemente la igualdad de hombres y mujeres en el mercado de trabajo, trasladándose a la sociedad ya como un hecho. Pero la realidad no se corresponde con esta imagen, persisten las diferencias entre los dos sexos en relación con la actividad de empleo ocupado en el sector de la construcción, con una clara desventaja para las mujeres.

A pesar de producirse cambios en la concepción de la educación universitaria, como un factor de empleabilidad y estabilidad laboral de la mujer en las disciplinas del Diseño, la Arquitectura y el Urbanismo, y en la lucha por la igualdad en el sector de la construcción, la realidad de las mujeres en el desarrollo profesional de las intervenciones sostenibles sigue con un avance mínimo que hay que paliar para el empoderamiento de las mujeres respecto a estos desequilibrios de género.

Centrándonos en el sector de la construcción desde la perspectiva de género en las intervenciones o conservaciones sostenibles en las edificaciones,

Feminismo/s 32, diciembre 2018, pp. 287-310 
para la Región de Murcia en concreto vemos cómo sigue existiendo una fuerte segregación ocupacional, aun teniendo, en los últimos años, una gran incorporación de la mujer a titulaciones de educación superior. Atendiendo a la tasa bruta de personas matriculadas en estudios universitarios, según el INE para la Región de Murcia, se observa que las posibilidades de formación para la mujer han mejorado. Contemplando el periodo de 2014 a 2017, visualizamos que hay un mayor índice de mujeres que realizan estudios universitarios de educación superior, pasando de 84.000 ocupadas por nivel de formación a 101.800 .

Ahora bien, ¿por qué si la mujer que accede al mundo laboral para realizar intervenciones sostenibles en edificación está más cualificada, no obtiene una mayor ventaja en la empleabilidad? Es importante tener en cuenta que el sexismo y la desigualdad de oportunidades en el sector de la construcción, ha determinado que históricamente las mujeres, como colectivo, hayan gozado de menos posibilidades de formación que los varones. Actualmente, la evolución es positiva y se ha conseguido el equilibrio. No obstante, y pese al mayor rendimiento educativo, dicha evolución no ha trascendido a la idea generacional de mejores sueldos, salarios, condiciones laborales y, en general, a mejorías del bienestar social. De hecho, estudios como el de Laura de Pablos y María Gil lo demuestran:

[...] la educación es relevante en los niveles de renta que se logran alcanzar por los individuos, más que en los cambios de estatus económico, mientras que la experiencia produce mayores rendimientos en los comienzos de la vida laboral. Además, cuando se analiza la fuente de rentas de los individuos según su nivel de educación, se aprecia que las rentas por cuenta ajena siempre son más importantes cualquiera que sea la edad del individuo analizado. Es más probable que los individuos jóvenes (18-30 años) con mayores niveles de educación no perciban renta alguna. (14)

Por ello, para que dicho reconocimiento universal de estatus formativo, económico social, y la consecuente eliminación de los estereotipos de género en las intervenciones sostenibles, no quede en una proclamación abstracta, deben conocerse las situaciones reales en las que se encuentran las personas con estudios superiores en el ámbito de la construcción (Chías 101-102). Estas medidas mostrarán si las mujeres pueden convivir como tales en condiciones de igualdad efectiva. Se trata de una filosofía de cambio actitudinal

Feminismo/s 32, diciembre 2018, pp. 287-310 
en la sociedad, basada en la ausencia de desigualdad respecto al concepto de las intervenciones sostenibles en las edificaciones, que se fundamenta en entender que las transformaciones y adaptaciones deben ser sobre el propio contexto y no sobre el individuo en sí mismo.

Si bien en el marco de la actual democracia no existen impedimentos para la participación en el ámbito de la construcción sostenible, consideramos que existen dificultades no explícitas que obstaculizan una plena participación femenina y dan explicación a que la participación de las mujeres sea aún minoritaria en todas las esferas del ámbito de la construcción, especialmente en las funciones de liderazgo en la obra. Es más, conocer nuestros derechos y las causas por las que se produce la inclusión o la exclusión en dicho ámbito, es el primer paso para tomar la iniciativa hacia una mayor participación social y, por ende, animar a demandar igualdad tanto en la toma de decisiones como en la obtención de un reconocimiento cultural y social de poder. Por ello, en cuanto a la toma de decisiones, consideramos necesario detenerse a deliberar sobre si la socialización de género en el ámbito de la conservación de edificios puede resultar beneficiosa para la mejora de la imagen urbana de la ciudad, mediante actuaciones transversales que eliminen los estereotipos de género y las expectativas educativas en el ámbito de la Arquitectura y de las Ingenierías, asentando un reconocimiento social de liderazgo y el avance de las mujeres por puro progreso. Como decía Lagarde:

[...] una mujer pionera no es la única, muchas de las que van detrás también son pioneras; todas estamos recién llegadas a muchos espacios, en cierta medida tenemos la marca de ser pioneras, muchas de nosotras sentimos no pertenecer a los espacios, que no son nuestros y no estamos cómodas porque cargamos subjetividad de pioneras. Tenemos que trabajar profundamente para eliminar la inseguridad de ser pioneras, el sentido de pertenencia, por una parte, y por otro, también tenemos que trabajar para reconocer la legitimidad de lo que hacemos. (251)

A su vez, este escenario nos permite partir de una reflexión clara: difícilmente podemos alcanzar a interpretar la problemática que plantea el ejercicio en las intervenciones sostenibles, si no lo deliberamos desde la imprescindible y compleja articulación de los derechos propios y ajenos de la ciudadanía (Chaux y Ruiz 52), el plano económico, la educación social y cultural (Arrazola y De Hevia 17-22).

Feminismo/s 32, diciembre 2018, pp. 287-310 
Derivado de lo anterior, pensamos que únicamente a través de una mejor información y educación paritaria a la ciudadanía, se podrá alcanzar el objetivo perseguido, trasladándose a las políticas energéticas relativas al ámbito de la construcción (Ramos et al. 18-27). Por tanto, en esta línea planteamos la hipótesis de que no basta con regular las intervenciones sostenibles del pasado, presente y futuro, sino que es necesario que todas las personas intervinientes comprendan íntegramente su significado y se pueda evolucionar hacia una integración horizontal y vertical en la profesión, diferencia pendiente de eliminar en materia de equidad entre el sexo femenino y el masculino.

\section{METODOLOGÍA Y ENCUESTA}

Además del análisis bibliográfico realizado, se ha utilizado una metodología cualitativa. Las reflexiones y aspectos comentados en las secciones anteriores han servido de base para la planificación del estudio empírico, con un cuestionario implementado por 37 participantes durante el mes de febrero de 2018.

La recogida de datos se ha obtenido de los/as egresados/as del Máster de Patología e Intervención en la Edificación, de la Universidad Católica de Murcia, desde el curso 2012-2013 hasta el curso 2016-2017. Los/as egresados/as tuvieron que responder a un cuestionario anónimo valorativo acerca de su criterio, para medir un constructo psicológico como opinión, sobre su percepción respecto a sus estudios de Posgrado y cómo lo vivenciaban, tanto respecto de su inserción laboral en el ámbito de la construcción como desde el punto de vista de la igualdad de género.

Ciertamente, esta metodología ofrece la posibilidad de que todos/as los/ as egresados/as del Máster citado sean interrogados/as exactamente con las mismas palabras y con la misma secuenciación de orden, ya que las preguntas por sí mismas pueden ser una fuente de error, en la medida que pueden desorientar al egresado o egresada.

Para la realización del correcto diseño del cuestionario anónimo valorativo se optó por el realizado con la Herramienta Google Drive, como instrumento para la recogida de datos para cubrir los aspectos clave del objeto de percepción a evaluar. Primeramente, se esbozó el primer borrador en el que se determinó la forma de las preguntas y la redacción de estas. A continuación, se procedió a la ordenación de las preguntas para facilitar su lectura,

Feminismo/s 32, diciembre 2018, pp. 287-310 
se determinó la escala de medida y se ajustó el primer borrador para esta investigación.

En este punto, ya definido el borrador de la encuesta, fue necesario realizar una prueba pretest para testar el borrador de encuesta, eliminar los posibles sesgos y detectar posibles problemas de comunicación. Tras la realización de una prueba pretest enviada al alumnado de la asignatura «Técnicas de intervención en el patrimonio edificado» en el Grado en Arquitectura de la UA, se procedió a incorporar las sugerencias de mejora de la encuesta. Básicamente, se eliminaron las preguntas dudosas de la encuesta inicial para intentar facilitar su comprensión y, además, simplificarla en tiempo.

En el sentido de la estructura, es de destacar que en el mail que se les enviaba a los/as egresados/as, después de presentarse las investigadoras, se solicitaba su colaboración para la investigación objeto de este artículo, presentándoles las líneas y objetivos más generales de la investigación y las instrucciones para su correcta implementación.

El/la encuestado/a debe leer detenidamente cada pregunta hasta el final y seleccionar la variable a contestar, y en caso de error, volver a escoger la respuesta válida. Es importante matizar que se insiste en que sus respuestas permanecerían en el anonimato.

Respecto al cuestionario diseñado ad hoc, en una primera parte se determinan variables sociodemográficas de identificación del alumnado (género, edad, estado civil y titulación de acceso), utilizando un formato de respuesta como lista de categorías, que tiene el objetivo de revelar la interrelación entre la percepción de qué aporta la perspectiva de género a las intervenciones sostenibles y sus características sociodemográficas. A continuación, cada egresado/a contesta 6 ítems que siguen o bien la modalidad de Escala de Likert con 4 respuestas válidas, desde bastante a nada, o bien la modalidad de respuesta dicotómica. Los objetivos de esta segunda parte de la encuesta son: evaluar y conocer la percepción de qué aporta la perspectiva de género a las intervenciones sostenibles desde el punto de vista tanto de las tasas de actividad laboral como de la igualdad de género. Para finalizar el mail de la encuesta, se procede a los agradecimientos a los/as egresados/as por la colaboración realizada.

En resumen, la encuesta posibilita focalizar el análisis del quehacer investigativo permitiendo un acercamiento más profundo y descriptivo sobre los

Feminismo/s 32, diciembre 2018, pp. 287-310 
desequilibrios de género en las intervenciones sostenibles, obtenido a través de la voz de los/as participantes egresados/as en el ámbito de la edificación.

Una vez recibidas todas las valoraciones de los/as egresados/as se procedió al análisis de la información ofrecida por dicha aplicación web, para la visualización de los resultados.

\section{RESULTADOS}

La investigación presenta la limitación de haberse desarrollado solamente en un espacio temporal concreto de la Región de Murcia, con la opinión de egresados/as de un sólo Máster dentro del ámbito de las carreras técnicas, impidiendo extender así los resultados obtenidos a un contexto territorial más amplio. De ahí que se considere únicamente válido a efecto de poder realizar reflexiones para la mejora del empoderamiento de la mujer respecto a los desequilibrios de género en su actuación laboral en las intervenciones sostenibles en las edificaciones, desde la disciplina del Diseño y la Arquitectura.

Dado que en 2017 la Región de Murcia estaba conformada por 101.800 mujeres $(50,47 \%)$ y 99.900 hombres $(49,53 \%)$ con estudios de educación superior, se considera que con respecto al porcentaje de egresados/as por sexo, la muestra estudiada conformada por 37 egresados/as ( 15 mujeres $-40,50 \%-y$ 22 hombres -59,50\%-) de los 44 posibles, podría calificarse como muestra total representativa.

Respecto a las franjas de edad, la mayor parte de los/as 37 egresados/as estaban en la comprendida entre los 25 y los 34 años. De forma ejemplificada y de manera concreta responden 11 egresados (29,73\%) y 12 egresadas $(32,43 \%)$. Cuando nos centramos en la franja de edad de los 35 a 44 años, la participación femenina es mínima, siendo sólo 1 egresada $(2,70 \%)$ frente a 8 egresados (21,62\%). Destacamos también una menor participación en las franjas de edad de los 45 a los 54 años, con tan sólo 1 egresado $(2,70 \%)$ y 1 egresada $(2,70 \%)$. En la franja de edad de los 55 a los 64 años, responden 2 egresados (5,41\%). Finalmente, sólo había 1 egresada (2,70\%) entre los menores de 24 años.

Si analizamos los datos estadísticos respecto al estado civil y sexo nos encontramos con un grupo bastante plural, lo que nos permite aproximarnos a la variedad de situaciones que viven los egresados y las egresadas 
actualmente. Básicamente, se encuentran solteros/as en el menor rango de edad, de 18 a 24 años, 1 egresada (2,70\%); seguidamente, en la franja de edad de los 25 a 34 años existen 8 egresados $(21,62 \%)$ y 8 egresadas $(21,62 \%)$; a continuación, de 35 a 44 años encontramos 2 egresados (5,41\%). Cuando nos centramos en los/as casados/as, el porcentaje disminuye, dado que existen 2 egresados $(5,41 \%)$ y 2 egresadas $(5,41 \%)$ en la franja de edad de los 25 a 34 años, y 5 egresados $(13,51 \%)$ y 1 egresada $(2,70 \%)$ en la de 35 a 44 años. No hay ningún egresado/a casado/a en la franja de menor edad. Por otro lado, observamos que solamente viven en pareja, 1 egresado $(2,70 \%)$ y 2 egresadas $(5,41 \%)$ en la franja de edad de los 25 a 34 años, y 1 egresado (2,70\%) en la franja de edad de 35 a 44 años. Cabe aclarar que no hay ni separados/as, ni viudos/as en la muestra.

Cuando nos centramos en el colectivo al que pertenecían los/as egresados/as antes de su entrada al Máster citado, el número de ingenieras de edificación (5) y de arquitectas técnicas (4) es similar al de arquitectas (4), siendo inferior en el caso de las ingenieras civiles (2). Respecto a los hombres, debemos decir que prefieren continuar sus estudios de grado en ingeniería de edificación (12), en arquitectura técnica (5), en ingenierías civiles (2) y en arquitectura (3).

Respecto a su incorporación a la vida laboral, el lastre de la crisis económica, la acentuación del paro y la precariedad de los contratos en este sector, hacen pensar que a priori exista más dificultad para encontrar trabajo. De hecho, los datos estadísticos demuestran que, de los 22 egresados que forman el colectivo masculino formado, trabajan 19 (86,36\%), sólo 1 (4,55\%) continúa estudiando, 1 egresado (4,55\%) trabaja en las tareas del hogar, y únicamente 1 de ellos (4,55\%) busca su primer empleo. Sin embargo, no se da la misma casuística para las 15 egresadas, 12 de ellas estaban trabajando $(80,00 \%), 2$ (13,33\%) estaban buscando todavía su primer empleo y 1 egresada $(6,67 \%)$ estaba parada.

Tras el análisis de estos datos concretos, nos preguntamos si tras la obtención de las mismas capacidades, el camino hacia la igualdad es ya una prioridad. Claramente vemos cómo se da una disminución en la segregación ocupacional por sexos, la cual podemos decir que se ha ido mitigando en los últimos años y que afortunadamente su posicionamiento positivo es cada vez más rápido puesto que en la estadística, la ocupación de las mujeres $(80,00 \%)$

Feminismo/s 32, diciembre 2018, pp. 287-310 
es ligeramente inferior a la de los hombres (86,36\%). Este hecho es debido, posiblemente, a que a día de hoy la mujer ingeniera o arquitecta esté cada vez más valorada y con un merecido rol dentro del sector de la construcción.

A continuación, se plantea la siguiente cuestión: «iTrabaja usted en el ámbito relacionado con las intervenciones en las edificaciones?» Cada una de las 3 egresadas (20,00\%) que no estaban trabajando contestó: «No, nunca he trabajado en el ámbito de las intervenciones sostenibles». Por otro lado, de las 12 egresadas (80,00\%) que están trabajando, 5 de ellas $(33,33 \%)$ contestaron «Sí, antes de realizar el máster», sorprendentemente sólo 2 de ellas (13,33\%) contestaron que «Sí, después de realizar el Máster», 6 de las egresadas $(40,00 \%)$ contestaron «No, pero he trabajado en el ámbito de la edificación». Finalmente, 1 de ellas $(6,67 \%)$ contestó que «No, pero he trabajado en el ámbito de la construcción. De estas respuestas, se intuye que las egresadas consideran que deben seguir estudiando un posgrado para facilitar su empoderamiento laboral y mejorar sus competencias en este ámbito, pudiendo competir con los roles laborales, todavía masculinizados a día de hoy. Respecto al sexo masculino, de los 19 egresados $(86,36 \%)$ que están trabajando, 8 de ellos $(36,36 \%)$ afirmaron que «Sí, después de realizar el Máster», 6 (27,27\%) contestaron que «Sí, antes de realizar el Máster», 5 egresados $(22,73 \%)$ respondieron «No, pero he trabajado en el ámbito de la construcción», y sólo 3 de los egresados $(13,63 \%)$ expresaron «No, nunca he trabajado en este ámbito de las intervenciones sostenibles». Queda claro que su especialización con el posgrado es un factor de peso que aporta un plus de valor para trabajar en este ámbito, de manera más clara que al sexo femenino.

En la siguiente pregunta: «Haber cursado el Máster en Patología e Intervención en la Edificación ¿Consideras que te ha abierto más posibilidades laborales relativas al sector de la construcción?» De los 22 egresados, $10(45,45 \%)$ contestaron que poco, 4 egresados (18,18\%) que bastante, 5 de ellos $(22,73 \%)$ que mucho y solamente 3 egresados $(13,64 \%)$ contestaron que nada. Por otro lado, de las 15 egresadas, 3 de ellas (20,00\%) consideraron que mucho, 2 (13,33\%) que bastante, 6 egresadas $(40,00 \%)$ que poco y sorprendentemente 4 de ellas (26,67\%) dijeron que nada. ¿Qué hace que perciban las mujeres, en mayor medida, como poco valiosos sus estudios de posgrado para la inserción laboral respecto a la opinión de sus compañeros varones? ¿Por qué esta invisibilización si sus capacidades y la formación recibida son

Feminismo/s 32, diciembre 2018, pp. 287-310 
similares? En realidad, consideramos que los datos reflejan que la no aceptación de su rol total en la sociedad hace que las egresadas que no trabajan sientan que no les han valido la pena sus estudios, percibiendo un rechazo del sector de forma más acusada que sus compañeros varones.

Respecto a la cuestión relacionada con los conocimientos adquiridos en el Máster, « ¿Considera que favorece el bienestar social y la mejora en las intervenciones del sector de la construcción desde el punto de vista de la restauración o rehabilitación?» De las 15 egresadas, sólo 2 de ellas $(13,33 \%)$ consideraron que mucho, $6(40,00 \%)$ manifestaron que bastante y 7 egresadas $(46,67 \%)$ opinaron que poco. Por el contrario, de los 22 egresados, 1 de ellos $(4,55 \%)$ consideró que nada, 2 egresados (9,09\%) que poco, 9 de los entrevistados $(40,91 \%)$ consideraron que bastante y 10 egresados $(45,45 \%)$ que mucho. Claramente existe una disparidad de percepción por género, donde los hombres consideran la igualdad efectiva y, las mujeres, la existencia de una clara desigualdad social.

La siguiente pregunta se formula de nuevo en relación al Máster, pero desde el punto de vista de visibilizar la igualdad de género en los estudios: «iConsidera que los conocimientos adquiridos en el Máster favorecen la igualdad de género en las intervenciones del sector de la construcción?» De nuevo, existe una disparidad de percepción, siendo positiva en el caso de los hombres, de los 22 egresados, 4 de ellos (18,18\%) consideró que nada, 4 egresados $(18,18 \%)$ que poco, $6(27,27 \%)$ consideraron que bastante y 8 egresados (36,36\%) que mucho. Y negativa en el caso de las 15 egresadas, de las cuales $3(20,00 \%)$ contestaron que nada, 4 de ellas (26,67\%) que poco, sólo 1 egresada $(6,67 \%)$ contestó que bastante y menos de la mitad, $7(46,67 \%)$ respondieron que mucho. Visiblemente, el rechazo en el trabajo a varias de ellas y el no estar trabajando en el ámbito de los estudios de posgrado, les ha hecho darse cuenta de que queda mucho camino por hacer. Sin embargo, la percepción del sexo masculino es que los roles de la sociedad van cambiando y se dirigen hacia una igualdad real y efectiva, siendo en realidad un primer paso para comprender y aceptar el fenómeno de empoderamiento femenino en el sector de la construcción, pues dicha comprensión es el primer paso para poder modificar las cosas.

Hecho que se corrobora con la última cuestión planteada: «En su experiencia laboral en el ámbito de las intervenciones del sector de la construcción

Feminismo/s 32, diciembre 2018, pp. 287-310 
desde el punto de vista de la restauración o rehabilitación ¿existe igualdad de oportunidades por género en los puestos de responsabilidad?» De las 15 egresadas, 11 de ellas (73,33\%) opinan que poco y sólo 4 egresadas (26,67\%) contestan que mucho. Pero paradójicamente, de los 22 egresados, 9 de ellos (40,91\%) opinan que mucho, 9 (40,91\%) consideran que bastante, 3 egresados $(13,64 \%)$ creen que poco y sólo 1 entrevistado $(4,54 \%)$ responde que nada. Es evidente que más de la mitad considera que hemos llegado a la igualdad efectiva. Sin embargo, los resultados indican que, en realidad, se trata de un espejismo que permitirá el cambio efectivo. De ahí que consideremos una mejora, a pesar de la pendiente y ardua tarea social que permita finalmente visibilizar y empoderar a la mujer.

\section{CONCLUSIONES}

Aunque en la Región de Murcia la igualdad en el desarrollo formativo de las mujeres en la disciplina del Diseño y la Arquitectura es una realidad, en el mercado de trabajo asociado a las intervenciones sostenibles sigue existiendo una clara discriminación laboral de género. De ahí que, respecto a los resultados de la investigación, el diseño, la elaboración e implementación de la encuesta en la asignatura del Máster de Patología e Intervención en la Edificación han ayudado a reflexionar sobre las complejas relaciones entre la educación universitaria, el empleo y el bienestar social para impedir que el objetivo de la ideología de género quede, una vez más, aplazado a los artífices de la cohesión y de la armonía social.

A continuación, se resumen las principales reflexiones que emanan de la praxis tanto en su vertiente teórica como en la empírica, resaltando las implicaciones que aporta el empoderamiento, real y deseado, de la perspectiva de género a las intervenciones sostenibles en España, enumerando las limitaciones del estudio empírico desarrollado y proponiendo líneas de acción para optimizar la incorporación de la mujer a distintas esferas de la vida laboral.

A pesar de la elevada presencia femenina en las titulaciones de arquitectura e ingeniería de edificación, las diferencias del estatus económico y los estereotipos de género en las intervenciones sostenibles en las edificaciones hacen que la desigualdad de género sea ineficaz e inadmisible, lo que conlleva a una clara segregación horizontal y vertical por discriminación de sexo. La

Feminismo/s 32, diciembre 2018, pp. 287-310 
encuesta ha puesto de manifiesto cómo las mujeres que luchan por acceder a un puesto laboral, en concreto en el sector de la construcción y para el ámbito de la rehabilitación y la restauración, dentro de la disciplina del Diseño y la Arquitectura, siguen tropezando con importantes barreras. Entre los principales factores responsables de frenar el desarrollo profesional de las mujeres en este ámbito de la construcción, destacan los estereotipos de género en este sector y la mayor presencia del varón dominante en el sistema laboral actual en este ámbito.

Es más, el problema de dicha ausencia es que para afrontar este reto hay que considerar la rehabilitación y restauración edificatoria actual en su propio contexto, teniendo en cuenta las complejas relaciones entre los estereotipos educacionales en el ámbito de la construcción. El currículo académico proporciona atribuciones para ejercer esa profesión en el ámbito de la sostenibilidad social, no sólo como un logro en el mundo de la construcción que empodere a las mujeres, sino también como un modo de afrontar en igualdad de condiciones las exigencias planteadas en las intervenciones sostenibles, desde la disciplina del Diseño y la Arquitectura.

El problema de fondo que trasciende es la necesidad de cambio hacia una intervención sostenible con perspectiva de género que exige transformaciones importantes, y en el centro de esas transformaciones está el cambio estructural del actual modelo de inserción laboral en el ámbito de la construcción, dado que sigue manteniendo invisibles a las mujeres respecto a la valoración social del trabajo que pueden llegar a ejercer.

Finalmente, dejamos aquí señalado que consideramos que la manera de restauración no es neutral, afectando a la sociedad desde el punto de vista de la obligación de mitigación del efecto del cambio climático, de ahí la necesidad de dirigir el trabajo profesional hacia el diseño de un perfil de empleabilidad, donde sea necesaria una mayor participación de dirección de obras en los niveles de toma de decisiones respecto a la rehabilitación sostenible, logrando una mayor igualdad de género en las posiciones de alta responsabilidad. Por tanto, a la luz de estas reflexiones, pensar en intervenciones sostenibles proporciona una oportunidad única para que ambos sexos trabajen en el ámbito de la construcción sin desigualdad, donde se puedan proponer una serie de medidas y criterios válidos y novedosos que permitan darles un nuevo uso y reducir el consumo energético.

Feminismo/s 32, diciembre 2018, pp. 287-310 


\section{REFERENCIAS BIBLIOGRÁFICAS}

Arrazola, María y José De Hevia. «Rendimiento de la educación en España: nueva evidencia de las diferencias entre hombres y mujeres». Papeles de Trabajo 21 (2001). 20 febrero 2018. <http://www.ief.es/docs/destacados/publicaciones/ papeles_trabajo/2001_24.pdf>

Barahona, Rodrigo, Joan Gratacós y Gotzon Quintana. Centros educativos transformadores. Ciudadanía global y transformación social. Barcelona: Oxfam Intermón, 2012. 15 febrero 2018. <https://www.oxfamintermon.org/es/ documentos/07/07/14/centros-educativos-transformadores-ciudadania-global-transformacion-social>

Barberá Ribera, Teresa, Sofía Estellés Miguel y Carlos Dema Pérez. «Obstáculos en la promoción profesional de las mujeres: El «techo de cristal»». Memorias del 3rd International Conference on Industrial Engineering and Industrial Management XIII Congreso de Ingeniería de Organización. Barcelona: Servicio de Comunicación y romoción Universidad Politécnica de Cataluña, 2009. 20 marzo 2018. <http://adingor.es/congresos/web/uploads/cio/cio2009/133142.pdf>

Chaux, Enrique y Alexander Ruiz. La formación de Competencias Ciudadanas. Bogotá: Ascofade, 2005. 20 febrero 2018. <https://laasociacion.files.wordpress.com/2015/11/la-formacion-de-competencias-ciudadanas.pdf>

Chías, Pilar. «Estudiantes de arquitectura ¿un ámbito de igualdad?». Feminismo/s 17 (2011): 91-103. 26 enero 2018. <https://ieg.ua.es/es/publicaciones/revista-feminismo-s/revista-feminismo-s.html>

Comisión Europea. Paquete de medidas sobre clima y energía hasta 2020, 2017. 26 enero 2018. <https://ec.europa.eu/clima/policies/strategies/2020_es>

De Pablos, Laura y María Gil. Movilidad de rentas y salarios bajo una perspectiva de género: el papel de la educación, 2006. 26 marzo 2018. <www.researchgate. net/publication/23547146>

Fernández, Ana María. La mujer de la ilusión. Pactos y contratos entre hombres y mujeres. Buenos Aires: Paidós, 1993.

Gil, María Rosario. La Dialéctica Mujer-Empleo: análisis de una realidad social, política, laboral y educativa. Málaga: Aljibe, 2005.

Infante, Margarita, Marisa Román y Joaquín Traverso. «La educación universitaria: un factor de empleabilidad y estabilidad laboral de la mujer en el sector de la construcción». Revista iberoamericana de educación 56 (2011): 1-11. 20 enero 2018.

Feminismo/s 32, diciembre 2018, pp. 287-310 
Infante, Margarita, Marisa Román y Joaquín Traverso. «El sector español de la construcción bajo la perspectiva de género. Análisis de las condiciones laborales». Revista de la Construcción 11 (2012): 32-43. 20 enero 2018. <http:// dx.doi.org/10.4067/S0718-915X2012000100004>

Instituto Nacional de Estadística. Encuesta de población Activa (EPA). Estadísticas publicadas. 15 de marzo de 2018. <http://www.ine.es/dyngs/INEbase/es/ operacion.htm?c=Estadistica_C\&cid=1254736176918\&menu=resultados\&idp $=1254735976595>$

Instituto de Estadísticas de Murcia (IEM). Estadísticas publicadas. <http//econet. carm.es/inicio/-/crem/sicrem/PU2050/sec7_cl.html>

Lamas, Marta. "Cultura, género y epistemología». Los estudios culturales en México. México: INI-CNA-FCE, 2003.

Lagarde, Marcela. Claves feministas para mis socias de la vida. Madrid: Horas y horas, 2005.

López, Mercedes, Ana Lisbona y Milagros Sáinz. «Mujeres ingenieras: percepciones sobre su vida profesional». Revista de psicología general y aplicada 57(2) (2004): 161-180. Marzo 2018.

Lucas, Javier de. «La lucha contra la discriminación en consolidación de derechos y garantías: los grandes retos de los derechos humanos en el siglo XXI». Seminario conmemorativo del 50 aniversario de la Declaración Universal de los Derechos Humanos. Madrid: Consejo General del Poder Judicial, 1999.

Navarro, Elena, Marisa Román y Margarita Infante. «Revisión internacional de estudios de barreras de carrera bajo la perspectiva de género en la industria de la construcción». Innovar 26 (2016): 103-118. 20 enero 2018. <http:// dx.doi.org/10.15446/innovar.v26n61.57169>

Parlamento Europeo y Consejo de la Unión Europea. Directiva 2010/31/UE del Parlamento Europeo y del Consejo relativa a la eficiencia energética de los edificios, 2010. 26 enero 2018. <https://eur-lex.europa.eu/legal-content/ES/ TXT/?uri=LEGISSUM\%3Aen0021>

Ramos, Ana, Alberto Gago, Xavier Labandeira y Pedro Linares. «The role of information for energy efficiency in the residential sector». Energy Economics 52 (2015): 17-29. 26 enero 2018. <https://doi.org/10.1016/j.eneco.2015.08.022>

Román, Marisa, Ana Ríos y Joaquín Traverso. «Barreras de género en el desarrollo profesional de mujeres técnicas de la construcción». Revista de la Construcción 12 (2013): 87-99. 15 marzo 2018. <http://www.redalyc.org/ articulo.oa?id=127628890009>

Feminismo/s 32, diciembre 2018, pp. 287-310 